\title{
Incidence, structure and morphological classification of abnormal sperm in the emu (Dromaius novaehollandiae)
}

\author{
Lizette du Plessis ${ }^{\mathrm{a}, *}$, John T. Soley ${ }^{\mathrm{b}}$

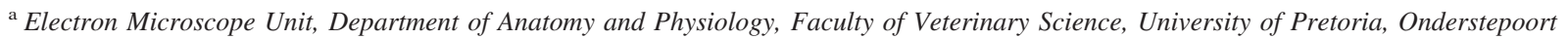 \\ 0110 , South Africa \\ ${ }^{\mathrm{b}}$ Department of Anatomy and Physiology, Faculty of Veterinary Science, University of Pretoria, Onderstepoort 0110, South Africa
}

Received 15 April 2010; received in revised form 14 June 2010; accepted 21 September 2010

\begin{abstract}
Little detailed information is currently available on the incidence and morphological characteristics of abnormal sperm in the emu (Dromaius novaehollandiae) and of ratites in general. This situation is further compounded by the lack of a uniform system for the morphological classification of avian sperm defects. Considering the important role that sperm morphology plays in the assessment of semen quality, a detailed description of avian sperm defects is of paramount importance. Based on morphological data provided by light and electron microscopy, a mean of $17.3 \%$ abnormal sperm was recorded in semen samples collected from the distal deferent duct of four adult emus during the middle of the breeding season. Four categories of defects were identified. Head defects (57.2\% of total defects) consisted of bent heads, macrocephalic heads, round heads and acephalic sperm. Zones of incomplete chromatin condensation and retained cytoplasmic droplets appeared to be implicated in head bending, while giant heads were often associated with multiple tails. Acephalic sperm revealed a complete tail devoid of a head which was replaced by a small spherical structure. Tail defects $(22.6 \%$ of total defects) were subdivided into neck/midpiece defects and principal piece defects. In the neck/midpiece region disjointed sperm were the exclusive defect noted and were characterized by the complete separation of the head and midpiece in the neck region but within the confines of the plasmalemma. Defects observed in the principal piece were subdivided into short tails, coiled tails and multiple tails. No conclusive evidence was obtained that tail coiling represented the 'Dag' defect. Biflagellate sperm were the most common form of multiple tails, demonstrating two complete tails with all the normal structural elements. Cytoplasmic droplets (13.9\% of total defects) were classified as a separate defect. The location and eccentric positioning of retained cytoplasmic droplets was similar to that described in ostrich sperm although the composition of the droplets differed markedly between the two species. A small percentage of sperm (6.3\% of total sperm defects) displayed multiple abnormalities. Based on these findings we propose a morphological classification for abnormal ratite sperm identifying head and tail defects, with additional categories for cytoplasmic droplets and multiple defects. Each category is further subdivided to reflect a range of specific defects within the category. It is envisaged that additional defects will be added to each category or that new categories may be added as future studies on the detailed morphology of avian sperm defects are completed.

(C) 2010 Elsevier Inc. All rights reserved.
\end{abstract}

Keywords: Emu; Dromaius novaehollandiae; Abnormal sperm; Incidence; Morphology; Classification

\footnotetext{
* Corresponding author. Tel.: +27 125298212.

E-mail address: lizette.duplessis@up.ac.za (L. du Plessis).
}

\section{Introduction}

In various parts of the world ratites such as ostriches, emus and rheas are farmed commercially for their skin, meat, fat and feathers. Although organised on a commercial basis, the industry is beset by a variety of 
production problems $[1,2,3]$, particularly in respect to reproductive performance, which severely hampers the economic viability of the industry. The ratite industry currently relies on natural reproduction, which is not always cost effective. In addition, the monogamous nature of ratites $[2,4]$ requires that a large number of males be kept for the sole purpose of breeding, adding an unnecessary financial burden to farming operations. Malecki et al [2] also point out that "natural mating hampers rapid genetic improvement because the genes of an elite male can be offered to only a few females." In order to address these constraints, it has been suggested that the ratite industry employ advanced reproductive technologies including the use of artificial insemination (AI) [2,4].

Successful AI programmes require proven methods for the collection, handling, storage and cryopreservation of semen, as well as the accurate assessment of male fertility. Sperm morphology plays an important role in the assessment of semen quality [5] and has been considered by Bertschinger et al [6] to be the most important parameter in assessing male fertility in the ostrich. While considerable information has been collected on normal sperm morphology of ratites and closely related species [7-11], little is known regarding abnormal forms in this group of birds. Various types of sperm abnormalities which result in a decrease in fertility and can lead to infertility have been identified in mammals, including man, and detailed descriptions of the defects including their ultrastructure have been documented [12-16]. There are a number of reports on the incidence of sperm abnormalities in birds, particularly in domestic poultry such as the fowl [17], turkey $[18,19,20]$, duck [21] and goose [22], as well as in ratites $[6,23,24,25]$ and exotic species $[26,27,28]$. However, the recording of the defects lacks conformity and the morphological description of the anomalies, confined mainly to light microscopic descriptions, suffers, with few exceptions [19,22,29,30,31],from a lack of ultrastructural detail. Although the incidence of sperm defects has been described in some ratites [23,24,25], only Malecki et al [23] presented a description of the defects observed. In contrast, Bertschinger et al [6] recognised five major defects in a study of 65 ostriches but did not report on the incidence of the defects.

With the exception of reports on the fine structure of cytoplasmic droplets in the ostrich $[29,30]$, the ultrastructural features associated with sperm defects in ratites have not been described. Such information may provide a better understanding of the origin, development and importance of sperm defects in this group of birds. The present study employed light and electron microscopy to examine the incidence and morphology of abnormal forms of sperm in semen samples from four emus and provides a comprehensive morphological description of defective sperm in this species. Based on these findings, a system for the uniform recording of ratite sperm abnormalities is proposed.

\section{Materials and methods}

Semen samples were collected during mid-breeding season from four clinically healthy, sexually active, adult emus following slaughter at a commercial abattoir. Samples were collected 60-100 min after the birds were slaughtered. Droplets of semen were gently squeezed from the distal ductus deferens into test tubes containing $2.5 \%$ glutaraldehyde in $0.13 \mathrm{M}$ Millonig's phosphate-buffer. Smears for light microscopy (LM) were prepared from the fixed cell suspensions, air-dried and stained with Wrights' stain (Rapidiff ${ }^{\circledR}$, Clinical Sciences Diagnostics, Johannesburg, South Africa). Smears from each bird were examined using a 100x oil immersion objective to evaluate sperm morphology and determine the incidence of sperm defects by counting the number of abnormal sperm present in a total of 300 cells.

Samples for transmission (TEM) and scanning electron microscopy (SEM) were fixed overnight in buffered glutaraldehyde. Using gentle centrifugation and re-suspension throughout, the samples were washed in Millonig's buffer, $\mathrm{pH} 7.4$ before post-fixation in similarly buffered $1 \%$ osmium tetroxide for one hour. After two subsequent washes in buffer, the samples were dehydrated through a graded ethanol series $(50,70,80$, 90, 96, 100\%). Samples for TEM were pelleted in Eppendorf tubes, embedded in epoxy resin, sectioned and stained with lead citrate and uranyl acetate before viewing in a Philips CM10 transmission electron microscope (Philips Electron Optical Division, Eindhoven, The Netherlands) operated at $80 \mathrm{kV}$. After the dehydration steps, the samples for SEM were incubated in hexamethyldisilazane (HMDS) for $30 \mathrm{~min}$ after which they were centrifuged, the supernatants discarded and the pellets resuspended in HMDS. A drop of this suspension was placed on a cover slip and allowed to air dry, after which it was sputter-coated with gold and viewed in a JEOL 840 scanning electron microscope (JEOL Electron Optics Instrumentation, Tokyo, Japan) operated at $8 \mathrm{kV}$.

Normal and abnormal sperm were expressed as a percentage of the total sperm count, while individual categories of abnormalities were expressed as a per- 
Table 1

Incidence of the various sperm defects observed in the present study expressed as a percentage of total sperm defects.

\begin{tabular}{|c|c|c|}
\hline Category & Region & Specific defect \\
\hline Head defects $^{1} 57.2 \%$ & Nucleus $57.2 \%$ & $\begin{array}{l}\text { Bent heads } 21.1 \% \\
\text { Macrocephalic } 18.3 \% \\
\text { Acephalic } 14.4 \% \\
\text { Round heads } 3.4 \%\end{array}$ \\
\hline Tail defects ${ }^{2} 22.6 \%$ & $\begin{array}{l}\text { Neck/midpiece } \\
7.2 \% \\
\text { Principal piece } \\
15.4 \%\end{array}$ & $\begin{array}{l}\text { Stump tails } 5.3 \% \\
\text { Coiled tails } 7.7 \% \\
\text { Multiple tails } 2.4 \%\end{array}$ \\
\hline \multicolumn{3}{|l|}{$\begin{array}{l}\text { Cytoplasmic } \\
\text { droplets }^{3} 13.9 \% \\
\text { Multiple defects } \\
6.3 \%\end{array}$} \\
\hline \multicolumn{3}{|c|}{$\begin{array}{l}1 \text { see paragraph } 3.2 .2 \text { for a detailed description of head defects. } \\
2 \text { see paragraph } 3.2 .3 \text { for a detailed description of tail defects. } \\
3 \text { see paragraph } 3.2 .4 \text { for a detailed description of cytoplasmic } \\
\text { droplets. } \\
{ }^{4} \text { see paragraph } 3.2 .5 \text { for a detailed description of multiple defects. }\end{array}$} \\
\hline
\end{tabular}

centage of total sperm abnormalities (Table 1). Measurements of the length and width of the various regions of the sperm cells were performed on scanning electron micrographs $(\mathrm{n}=40)$, while measurements comparing the head lengths of normal $(n=370)$ and apparently diploid sperm (giant heads; $n=46$ ) were obtained using light micrographs. Both sets of measurements were processed using the Soft Imaging System iTEM software (Olympus, Münster, Germany) and expressed as the mean $\pm \mathrm{SD}$.

\section{Results}

\subsection{Normal Sperm}

In the four birds studied, $83 \%$ of the sperm displayed the normal morphological features previously described for the emu $[8,32]$. The sperm were typically filiform in shape (Figs. 1 and 2) with a slender tapering head capped by a small conical acrosome (Fig. 1 insets a,b). The acrosome was short, measuring $2.3 \pm 0.3 \mu \mathrm{m}$ in length and $0.4 \pm 0.05 \mu \mathrm{m}$ wide at its point of termination. The cylindrical nucleus was $9.5 \pm 0.4 \mu \mathrm{m}$ long and $0.78 \pm 0.05 \mu \mathrm{m}$ wide at the base. The surface of the head, particularly of the acrosome, was smooth when viewed by SEM (Fig. 1 inset b). Longitudinal sections of the sperm examined by TEM confirmed that the apical tip of the highly condensed nucleus extended beneath the acrosome (Fig. 1 inset a). A narrow subacrosomal space occupied by flocculant, moderately electron-dense material lay between the nucleus and the acrosome. No perforatorium was present. A short proximal centriole lay below the base of the nucleus at right

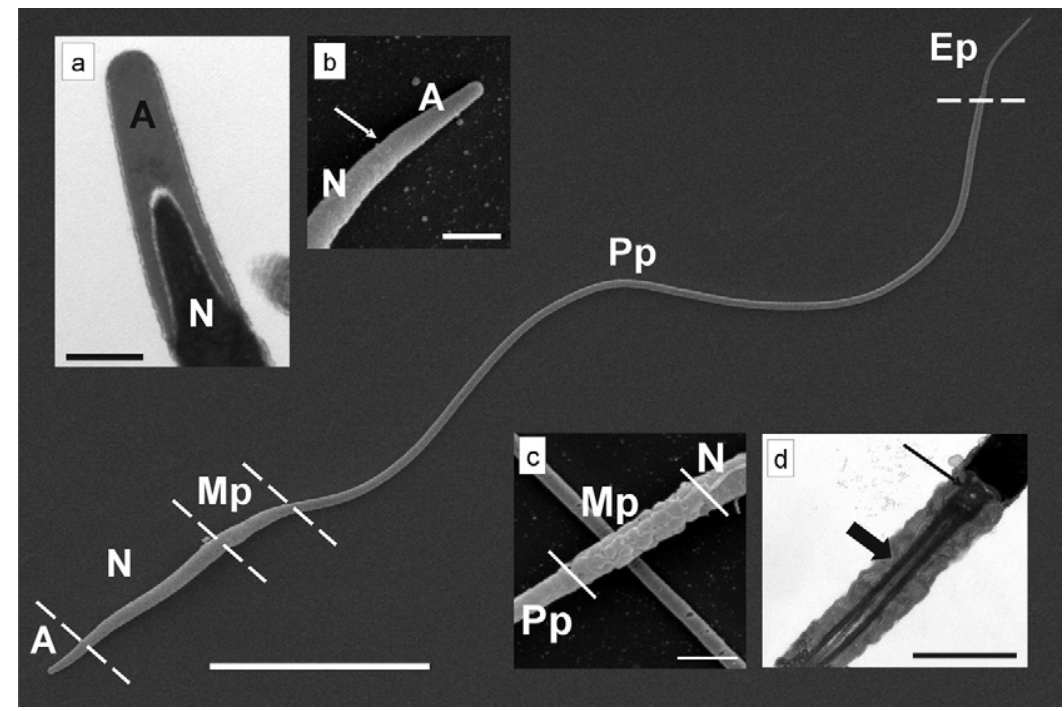

Fig. 1. SEM of a normal emu sperm illustrating the chief structural components, namely the head composed of the acrosome (A) and nucleus (N), the midpiece (Mp), principal piece (Pp) and endpiece (Ep). Bar $=10 \mu \mathrm{m}$. Inset (a). Section through the acrosome (A) covering the tapered tip of the nucleus (N). TEM. Bar $=1 \mu \mathrm{m}$. Inset (b). The conical acrosome (A) reveals a smooth surface and a discrete junction $(\rightarrow)$ with the nucleus $(\mathrm{N})$. SEM. Bar $=1 \mu \mathrm{m}$. Inset (c). SEM of the midpiece $(\mathrm{Mp})$ illustrating the mitochondrial sheath, nucleus $(\mathrm{N})$ and principal piece $(\mathrm{Pp})$. Bar $=$ $1 \mu \mathrm{m}$. Inset $(\mathrm{d})$. TEM of the midpiece showing the mitochondrial sheath arranged around the proximal $(\rightarrow)$ and distal $(\rightarrow)$ centrioles. The distal centriole runs the full length of the midpiece. Bar $=1 \mu \mathrm{m}$. 


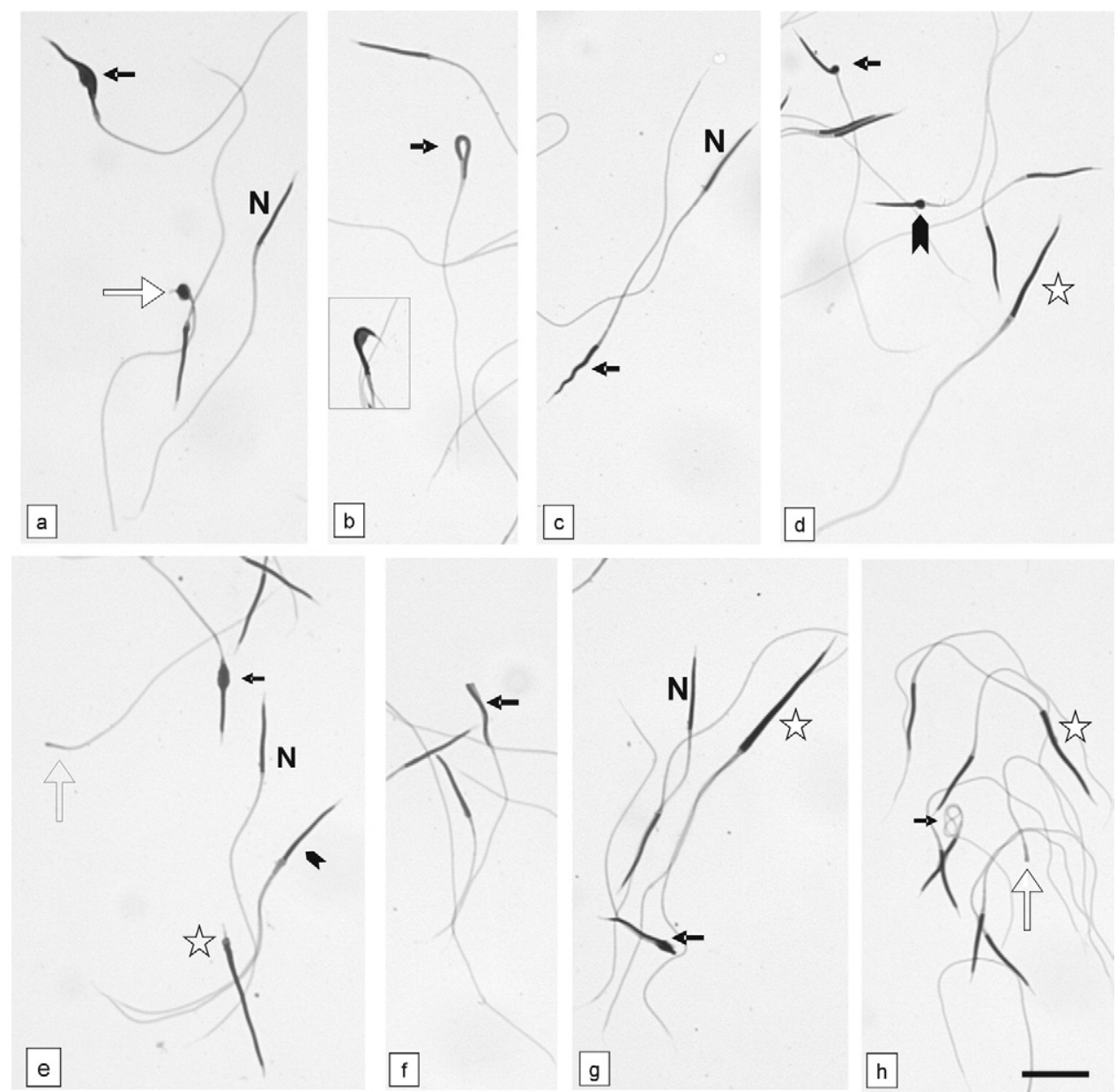

Fig. 2. Light micrographs illustrating morphologically normal sperm $(\mathrm{N})$ and various sperm defects. (a) Large cytoplasmic droplet covering the head base and proximal midpiece $(\rightarrow$ ) and round-headed sperm $(\hookrightarrow)$. Note the acrosome-like structure associated with the round-headed sperm. (b) Looped sperm head $\leftrightarrow$ ). Inset: Bent head with cytoplasmic droplet. (c) Spiralled head $\leftrightarrow$ ). (d) Kinked head $\leftrightarrow$ ), small cytoplasmic droplet $(\leftrightarrow)$ and a giant sperm with double tail $(\downarrow)$ ). (e) Large cytoplasmic droplet $\leftrightarrow$ ), acephalic sperm $(\hookrightarrow)$ ), sperm with normal-sized head and double tail $(\leftrightarrow)$ and giant sperm with a short tail $(\hbar)$. (f) Disjointed sperm $(\rightarrow)$. (g) Giant sperm with double tail ( $\downarrow$ ). Note the comparative size difference

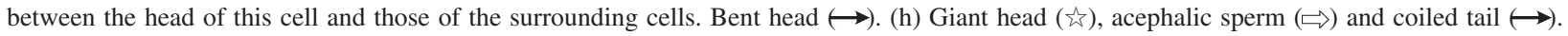
Bar $=10 \mu \mathrm{m}$.

angles to the long distal centriole that extended the full length of the mid-piece (Fig. 1 inset d). The implantation fossa was poorly developed and formed a shallow concave depression or series of smaller depressions. The midpiece, $3.3 \pm 0.5 \mu \mathrm{m}$ long and $0.5 \pm 0.04 \mu \mathrm{m}$ wide, was not well defined on LM but could usually be distinguished on SEM by the presence of mitochondrial profiles (Fig. 1 inset c). The midpiece consisted of approximately 40 closely packed, rectangular mitochondria with parallel cristae (Fig. 1 inset d), although some round forms were observed. The annulus demarcated the midpiece from the principal piece with its ribbed fibrous sheath and which terminated in a short endpiece $(3.6 \pm 0.6 \mu \mathrm{m})$. The total length of the sperm was $64.0 \pm 2.0 \mu \mathrm{m}$.

\subsection{Abnormal Sperm}

\subsubsection{Incidence}

A mean value of $17 \%$ morphologically abnormal sperm was reflected in the samples examined. These defects were divided into four broad categories, namely, head defects (57.2\%), tail defects (22.6\%), multiple defects $(6.3 \%)$ and cytoplasmic droplets (13.9\%) (Table 1, Fig. 2).

\subsubsection{Head defects}

Most abnormalities occurred in the head region of which four forms, namely, bent heads, macrocephalic heads, round heads and acephalic sperm, were identi- 

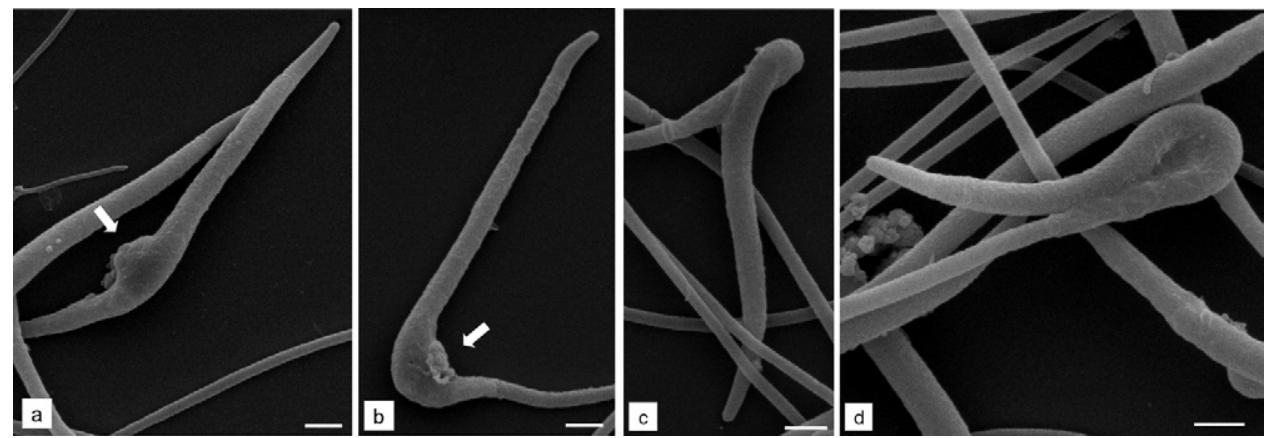

Fig. 3. A series of scanning electron micrographs illustrating various forms of head bending including (a) a gentle bend, (b) a $90^{\circ}$ bend, (c) kinking or twisting of the head near the base and (d) a looped head bend. Note how the attached cytoplasmic droplet $(\Rightarrow)$ in (a) and (b) determines the direction of the head bend. The plasmalemma appears to enclose the bent portion of the head in the looped form of the defect seen in (d). Bar $=1 \mu \mathrm{m}$.

fied (Figs. 2, 3, 4, 5 and 6). Various forms of head bending could be distinguished ranging from simple bending (gentle to acute bends), to looping, kinking (which manifest as a single twist of the head, generally towards the base), coiling (in most instances the formation of a single coil) and spiralling. Spiralled heads presented a corkscrew appearance and was the least frequent head defect noted. Bent heads were often associated with retained cytoplasmic droplets. The eccentric positioning of the droplets effectively determined the direction of the bend (Figs. 2a,b and 3a,b). Head bending was also frequently associated with defective or incomplete chromatin condensation, particularly towards the base of the nucleus. In such instances a variably-sized gap in the karyoplasm filled with flocculent material was present in the immediate vicinity of the bend (Fig. 4a,b,c).

Macrocephalic heads were markedly larger than normal heads (Fig. 2d,e,g,h), measuring $17.86 \pm 1.87 \mu \mathrm{m}$ in length. The average length of normal heads was $11.02 \pm 0.78 \mu \mathrm{m}$. These giant heads were associated with either a single tail or multiple tails. Round-headed sperm were occasionally noted (Figs. 2a and 5a). The heads were spherical in shape, varied in size, and in some instances revealed a projection resembling the acrosome. Sections of round-headed sperm showed a round nucleus with condensed chromatin (Fig. 5b). Acephalic sperm were easily identified on LM and SEM (Figs. 2e,h and 6). The cell consisted of a complete flagellum (midpiece, principal piece and endpiece) of normal dimensions and morphology but devoid of a head, which was replaced by a small spherical structure. No acrosome defects were observed in the material studied.

\subsubsection{Tail defects}

Tail abnormalities were subdivided into neck/midpiece and principal piece defects. Only one defect,
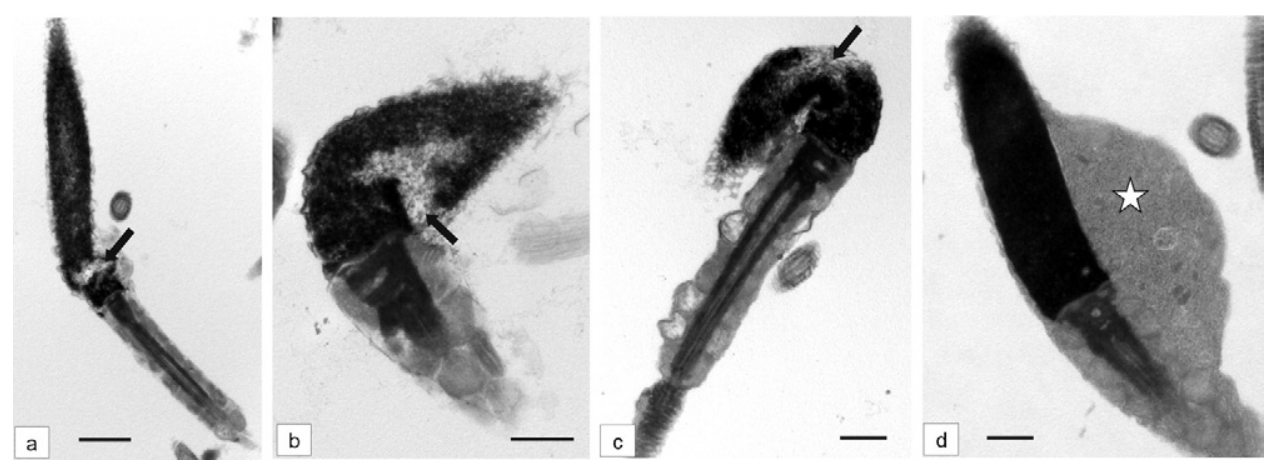

Fig. 4. Longitudinal sections of various forms of head bending (a-c) and a retained cytoplasmic droplet (d). The degree of bending in Figs. a, b and c is equivalent to that illustrated in Fig. 3 a, b and d respectively. Note the large, poorly condensed region of chromatin $\leftrightarrow$ ) near the nuclear base associated with the bending. The cytoplasmic droplet illustrated in (d) is eccentrically positioned, covers the base of the head and part of the midpiece, and is composed mainly of fine, homogenous material (论). (a) Bar $=2 \mu \mathrm{m}$; (b-d) Bar $=1 \mu \mathrm{m}$. 

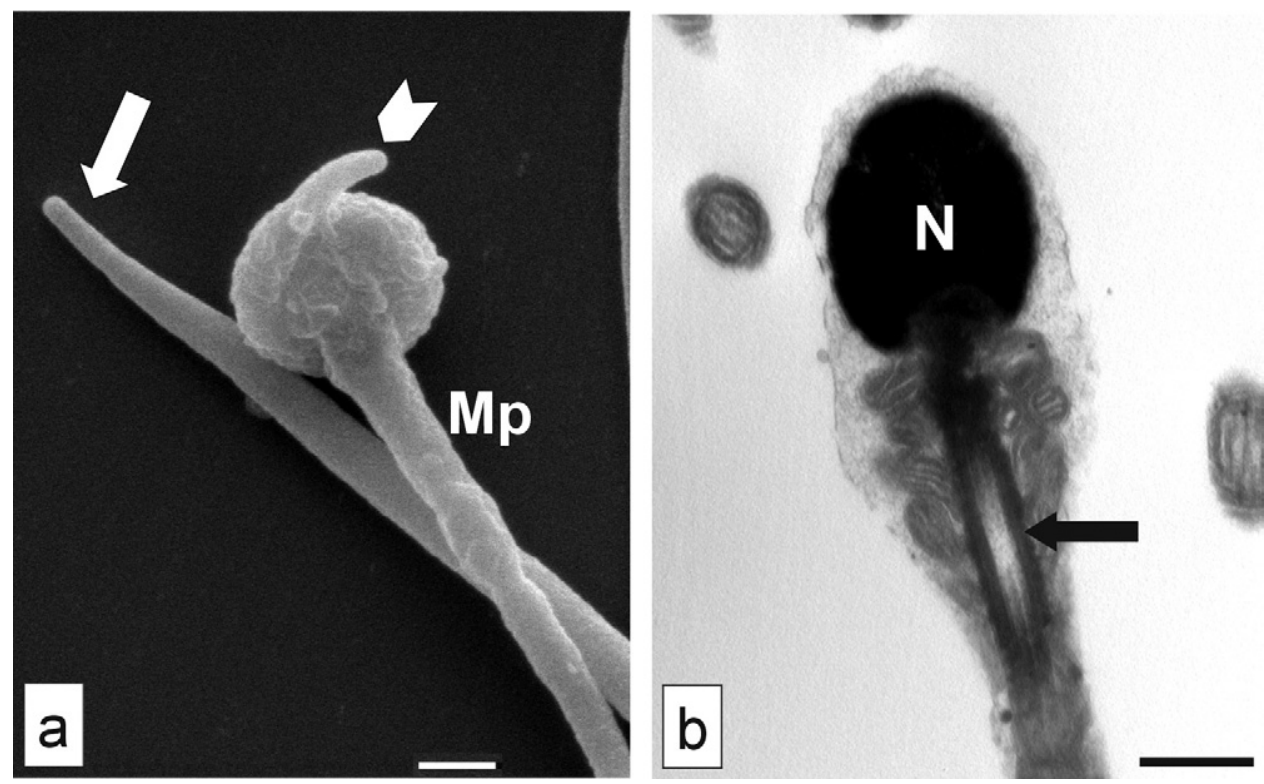

Fig. 5. Round-headed sperm. (a) The head consists of a round mass of material attached to the proximal extremity of the midpiece (Mp) and from which a structure resembling the acrosome $(\Sigma)$ emerges. Compare the presumptive acrosome with that of a normal sperm head on the micrograph $(\Rightarrow)$. See also Fig. 2(a). SEM. Bar $=1 \mu \mathrm{m}$. (b) Longitudinal section of a round-headed sperm showing similar morphology to the cell in Fig. 5(a), with the exception of the presumptive acrosome. Note the round shape of the nucleus $(\mathrm{N})$ and the condensed nature of the chromatin. The distal centriole $(\rightarrow$ is enclosed by the mitochondrial sheath. TEM. Bar $=1 \mu \mathrm{m}$.

disjointed sperm, was observed in the neck/midpiece. This defect was characterized by the complete separation of the head and midpiece in the neck region but within the confines of the plasmalemma (Figs. $2 \mathrm{f}$ and 7 ). The specific point of separation appeared to occur at the implantation fossa. On LM and SEM the defect appeared as a tight (complete) reflection of the head with the base of the head and the midpiece lying next to each other, the cell thus appearing disjointed.

Abnormalities of the principal piece were subdivided into stump (short) tails (Fig. 2e,f), coiled tails (Fig. 2h) and multiple tails (Figs. 2d,e,g and 8). Short-

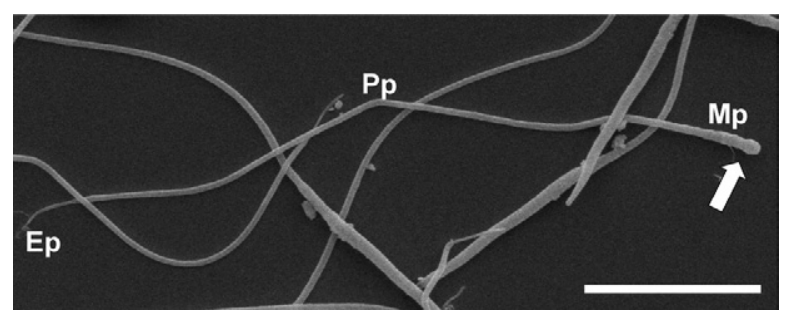

Fig. 6. SEM illustrating the full length of an acephalic sperm. The sperm neck is represented by a small gently swollen structure $(\Rightarrow)$. The tail reveals all the normal components in their correct proportions, namely the midpiece $(\mathrm{Mp})$, principal piece $(\mathrm{Pp})$ and endpiece (Ep). Note the obvious absence of the head. Bar $=5 \mu \mathrm{m}$. tailed sperm displayed a range of under-length flagella, from an extremely shortened tail typical of the mammalian stump tail defect [12] to a flagellum approximately half the normal length. Coiled tails demonstrated one or more tight coils of the principal piece whereas multiple-tailed sperm exhibited two or more flagella. Tail coiling appeared to involve only the principal piece which was thrown into two or more coils. The coils sometimes displayed a figure of eight configuration (Fig. 2h) and in some instances appeared on SEM to be enclosed within the plasmalemma. However, due to the limited number of cells with coiled tails in the samples studied, missing or translocated elements of the axoneme, indicative of the 'Dag' defect, were not observed on TEM.

On SEM, some sperm with double tails displayed a double midpiece (Fig. 8a), whereas in others only an apparently single midpiece was evident. However, longitudinal and transverse sections of the midpiece clearly revealed the presence of two centriolar complexes with their respective mitochondrial sheaths (Fig. $8 \mathrm{~b}, \mathrm{c})$. The double centriolar complexes were associated with duplicated elements of the connecting piece and, due to the limited space available at the base of the head, attached abaxially via shallow, twin implantation fossae (Fig. 8b). The nuclear base was often abnormally 

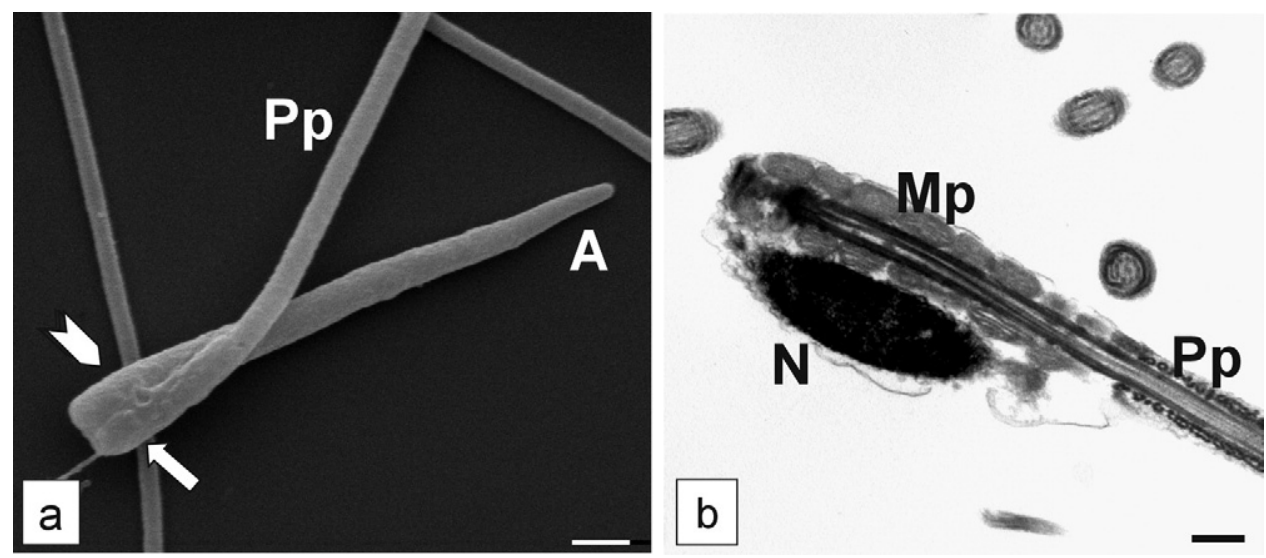

Fig. 7. Disjointed sperm. (a) SEM. Note the parallel positioning of the base of the head $(\square)$ and the completely reflected midpiece $(\rightarrow)$. The head base and midpiece appear to be attached by the plasmalemma. Acrosome (A), principal piece (Pp). Bar $=1 \mu \mathrm{m}$. (b) TEM of a presumptive disjointed sperm with similar parallel positioning of the base of the head $(\mathrm{N})$ and the midpiece $(\mathrm{Mp})$ as illustrated in (a). Both the head and midpiece lie within the confines of the plasmalemma. Principal piece $(\mathrm{Pp})$. Bar $=1 \mu \mathrm{m}$.

attenuated at the periphery to accommodate the twin tails. The twin centriolar complexes and attendant structures, as well as the components of the midpiece and principal piece (axoneme, mitochondrial sheath, annulus and ribbed sheath) generally appeared normal, although the mitochondrial sheath displayed
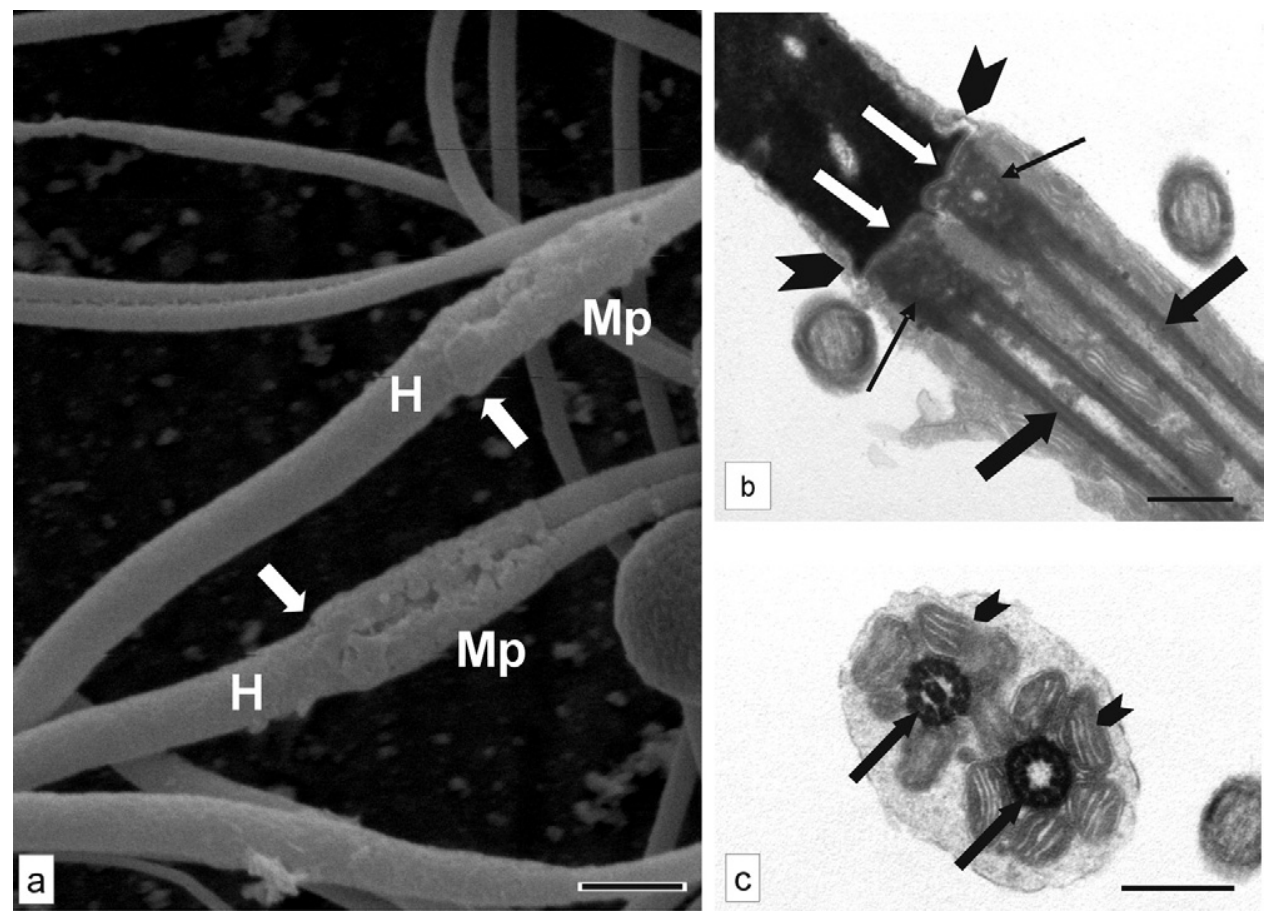

Fig. 8. (a) SEM showing the double tails of two biflagellate sperm emerging from the head base (H). The double midpieces (Mp) appear to be separate. Note that the point of midpiece attachment extends beyond the width of the head base $\leftrightarrow$ ). Bar $=1 \mu \mathrm{m}$. (b) TEM of a biflagellate sperm. The two centriolar complexes attach to the nuclear (head) base in the normal fashion via the connecting piece and associated proximal centrioles $\leftrightarrow$ ). Note how the nuclear base extends peripherally $(\rightarrow)$ to accommodate the twin tails $(\rightarrow)$. Also note the shallow implantation fossae $(\Rightarrow)$ and the single layer of mitochondria between the centriolar complexes. Bar $=1 \mu \mathrm{m}$. (c) Transverse section through the proximal midpiece of a biflagellate sperm. The two midpieces are attached and show the normal arrangement of the distal centriole $\rightarrow$ ) surrounded by the mitochondrial sheaths $(\rightarrow)$. The lumen of one centriole appears empty, while the other contains a pair of microtubules. Bar $=1 \mu \mathrm{m}$. 
only a single row of mitochondria between the two centriolar complexes.

\subsubsection{Cytoplasmic droplets}

Cytoplasmic droplets (Figs. 2a,b,d,e, 3a,b and 4d) varied in size, appeared bulbous and were mostly eccentrically positioned. They were located either at the base of the head or covered both the head base and proximal section of the midpiece. The droplets were contained by the plasmalemma and consisted of a homogeneous, finely granular cytoplasmic matrix with an occasional mitochondrion and/or vesicle (Fig. 4d).

\subsubsection{Multiple defects}

A small percentage of sperm displayed more than one abnormality simultaneously. Multiple defects included, for example, bent heads with multiple, short or coiled tails, multiple tailed sperm with droplets or macrocephalic heads with droplets and/or multiple tails (Fig. 2d,g).

\section{Discussion}

\subsection{Incidence of sperm defects}

Conflicting information has been presented concerning the presence of abnormal sperm in avian semen. While Gee et al [33] noted that a variety of unusual sperm types are common in semen in some species, Blanco et al [34] reported that sperm pleomorphisms are uncommon in fresh semen samples. Abnormal sperm are commonly identified in ratite semen with the incidence ranging from approximately $5 \%$ [25] to $17 \%$ [24] and 25\% [6] in the ostrich. A similar incidence of $17 \%$ abnormal sperm was observed in the emu (present study) although a much lower percentage of abnormalities (in the region of 2\%) was reported by Malecki et al [23] in ejaculates of the same species. Alkan et al [20] recorded a figure of $17 \%$ morphologically abnormal sperm in turkey semen. Rozenboim et al [25] and Bertschinger et al [6] both reported that more abnormal forms were detected in ostrich semen at the beginning of the breeding season with the least number of abnormalities being found midseason. In the Northern pintail duck, the percentage normal sperm in semen samples collected mid-season is high (82.1\%) [21], which corresponds to the figure of $83 \%$ normal sperm obtained in the present study of emu samples collected in mid-season.

\subsection{Structural features of specific abnormalities}

\subsubsection{Head defects}

Bent heads were readily identified by light and electron microscopy with both techniques reflecting the full range of the defect, from gentle bends to more prominent forms such as looping, spiralling and twisting of the head. In the majority of bent heads observed by TEM, the chromatin in the vicinity of the bend was poorly compacted or even absent, particularly near the base of the head. Although bent heads have been observed in other avian species by LM [20,21,22,35], this study provides the first evidence that defective chromatin condensation during spermiogenesis may be a contributory factor in the development of this defect. The significance of this observation is strongly supported by an in-depth study in humans suggesting that "sperm head defects may in part be due to reduced nuclear compaction" [36]. In addition, the observation that cytoplasmic droplets (see below) are often associated with head bending also implicates inconsistency in the removal of residual cytoplasm during spermiation in the formation of bent heads. Distention of the plasmalemma induced by the use of hypotonic extender is also reported to cause head bending (towards the base of the nucleus) as well as various degrees of tail bending [37].

Macrocephalic sperm were almost as prevalent as bent heads and often associated with multiple tails. Sperm with giant heads have previously been reported in the emu [23] where they formed a large percentage of total sperm abnormalities. Similar sperm have been observed in large numbers in Houbara bustard semen, the nucleus measuring up to twice the mean length of the nucleus of normal sperm [26]. Based on this obvious size differential and on the intense diamidino-phenylindole (DAPI) staining seen throughout the nucleus, it was suggested that "these spermatozoa contained more than the haploid content of genetic material and that some were actually diploid." [26]. A similar situation is indicated in the present study where the giant heads identified by LM measured approximately 1.5 times the length of normal sized sperm heads. The linear measurements of normal sperm obtained in this study correlated well with the dimensions reported by Baccetti et al [8] and Jamieson [32].

The incidence of round-headed sperm in the present study was low and it appears to be an uncommon defect in birds. Such sperm display an apparently normal acrosome capping a condensed, but round nucleus. Their ultrastrucural features seem to indicate that they are the product of defective spermiogenesis, specifically involving the failure of nuclear elongation. It would be interesting to determine whether this defect is the product of defective formation of the circular manchette which has been credited with initiating and 
maintaining nuclear elongation in a number of avian species, including the ostrich [38].

A significant finding was that of acephalic sperm in all of the emu samples studied and which have not previously been reported in ratite semen. Łukaszewicz et al [17] and Ferdinand [22] both illustrate an abnormal sperm in fowl and goose semen respectively which appears remarkably similar to the acephalic sperm observed in the present study, but which is referred to as a spermatid (fowl) and a small round-headed sperm (goose). In mammals, acephalic sperm are known to originate during spermiogenesis through a malformation in the neck region of the spermatid tail which prevents connection of the sperm head and flagellum $[14,39,40]$. These cells display a normal axoneme, although an abnormal knob-like grouping of displaced mitochondria is often seen at the anterior aspect of the cell. This phenomenon often results in these cells being incorrectly categorised as microcephalic on smears [14]. LM and SEM preparations confirmed the knoblike anterior aspect of emu sperm displaying this defect although the characteristic ultrastructural features could not be confirmed by TEM, again due to the relative scarcity of the defect. The fact that detached heads (morphologically normal heads without a tail attached) were almost entirely absent in smears and SEM preparations studied would confirm, as the literature implies [40], that acephalic sperm originate during spermiogenesis and are not the product of separation of the head and tail following spermiation or the result of sample processing.

\subsubsection{Tail defects}

Disjointed sperm represented the only neck/midpiece defect observed in the emu. This unique anomaly was characterised by complete separation of the head and midpiece, which lay adjacent and parallel to each other within the confines of the plasmalemma. To what extend the shallow implantation fossa typical for emu sperm and also reported in ostrich sperm [10], may be a predisposing factor in causing this defect, remains unknown. Sperm with bent necks [17,41], also referred to as crooked-necked [42] or broken-necked sperm [43], have been reported in poultry and other avian species and bear some morphological similarity on LM to the disjointed sperm seen in this study. However, crooked-necked sperm appear to be an acute form of midpiece bending and are not associated with discrete separation of the head and midpiece. It should be noted that some of the sperm identified by LM as 'disjointed' may in fact represent acute bending (reflex) of the midpiece. However, difficulty in resolving the subtle difference between an acute bend in the neck/proximal midpiece region and actual separation of the head and neck/midpiece at the light microscopic level led to the creation of a single category for disjointed sperm. Allowances are however made in the proposed classification of ratite sperm defects for midpiece bending (see Table 2).

Tail abnormalities manifested as short or stump tails, coiled tails and multiple tails. Tail defects are important as they potentially influence the motility of the sperm [14]. Stump tails have been observed in a number of mammalian species [12]. The incidence of this defect is generally low but when present, has serious implications for fertility [12]. Stump tails formed only a small proportion of total tail defects in the emu. On LM and SEM, the form and arrangement of some coiled tails were reminiscent of the 'Dag' defect described in mammals $[15,44,45]$ and also reported to occur in the ostrich [6]. The 'Dag' defect is characterised by varying degrees of tail coiling within an intact plasma membrane as well as disruption and translocation of elements of the axoneme [15]. However, these specific features could not be confirmed by TEM in the present study due to the relative scarcity of the defect. Multiple tails have been described in humans [15,46], bulls [47], geese [22] and in the duck [48]. The biflagellate sperm observed in the emu displayed similar morphological characteristics to those described in the drake [48]. Lindsay and co-workers [26] reported on giant heads occasionally associated with biflagellated sperm in the Houbara bustard. In this study, double tails were associated with either normal or giant sized heads. This defect is believed to be caused by defective spermatid development [46]. The stump tail defect, 'Dag' defect and multiple tails have been reported to adversely affect fertility $[6,12,13,49]$. The term 'bent sperm' has been used to describe tail bending in poultry involving both the midpiece and principal piece $[31,37]$. This defect, together with associated anomalies such as distention of the plasmalemma, mitochondrial swelling and tail coiling has been reported to result from adverse osmotic conditions and the effects of preand post-freezing treatments on fowl and turkey sperm. None of these anomalies was observed in the present study, and tail coiling formed only a small percentage of total sperm defects.

\subsubsection{Cytoplasmic droplets}

Cytoplasmic droplets are known to constitute a high proportion of the sperm abnormalities seen in emu semen samples [23]. This defect was consistently observed in the 
Table 2

A proposed classification for ratite sperm abnormalities based on observations from the present study and documented information on ratites and other avian species.

\begin{tabular}{|c|c|c|c|}
\hline Category & Region & Specific defect & Description \\
\hline \multirow[t]{8}{*}{ Head defects } & Acrosome $^{1}$ & Detached & Acrosome missing $[20,22]$ \\
\hline & & Deformed & $\begin{array}{l}\text { Includes unusual shapes, such as small, knob-like, round or comma-shaped } \\
\text { as well as swelling (also described as bulbous) of the acrosome }[20,22,35]\end{array}$ \\
\hline & Nucleus & Bent & $\begin{array}{l}\text { Any form or degree of bending of the nucleus, including looping, coiling, } \\
\text { kinking and spiraling. Also referred to as knotted, twisted and hooked } \\
{[18,20,22]}\end{array}$ \\
\hline & & Round head & Spherical-shaped nucleus \\
\hline & & Macrocephalic & $\begin{array}{l}\text { Head larger than the average; also described as giant, enlarged or large } \\
\text { heads }[23,26,27,35]\end{array}$ \\
\hline & & Microcephalic ${ }^{1}$ & Head smaller than the average; also described as under-sized [27] \\
\hline & & Acephalic & $\begin{array}{l}\text { Normal neck, midpiece and tail but no head. No detached heads observed } \\
\text { in sample }\end{array}$ \\
\hline & & Detached head $^{1}$ & $\begin{array}{l}\text { Total detachment of the head from the tail but associated with loose tails; } \\
\text { also described as loose heads }[6,20,22]\end{array}$ \\
\hline \multirow[t]{7}{*}{ Tail defects } & Neck/midpiece & Disjointed & $\begin{array}{l}\text { The nucleus lies parallel to the midpiece but separated from the latter, } \\
\text { with both components enclosed by the plasmalemma }\end{array}$ \\
\hline & & Deformed $^{1}$ & $\begin{array}{l}\text { Includes swelling, thickening, vacuolization and partial or complete aplasia } \\
\text { of the mitochondrial sheath }[6,20,22,35]\end{array}$ \\
\hline & & Bent $^{1}$ & $\begin{array}{l}\text { Includes varying degrees of bending, midpiece reflex or kinking in this } \\
\text { region; also referred to as crooked- or broken-necked }[6,20,22,42,43]\end{array}$ \\
\hline & Principal piece & Stump & Visibly shorter tail than normal [22] \\
\hline & & Multiflagellate & Two or more flagella present \\
\hline & & Coiled & $\begin{array}{l}\text { Includes 'Dag' defect. Also referred to as curled, knotted, bent, rolled } \\
{[6,27,35]}\end{array}$ \\
\hline & & Loose tail ${ }^{1}$ & $\begin{array}{l}\text { Total detachment of the tail from the head, in association with detached } \\
\text { heads }[20,22]\end{array}$ \\
\hline Cytoplasmic droplets & & & $\begin{array}{l}\text { The presence of remnant cytoplasmic material; may cover the base of the } \\
\text { nucleus, the midpiece or both regions }\end{array}$ \\
\hline Multiple defects & & & Combination of two or more defects from different regions of the sperm \\
\hline
\end{tabular}

\footnotetext{
${ }^{1}$ None observed in the present study, but described in other avian species.
}

present study, with the droplets varying in size. The location and eccentric orientation of these structures were similar to those described for the ostrich $[29,30]$. However, the composition of the droplets differed markedly between the two species with those of the ostrich containing lipid-like droplets and membranous structures while those of the emu generally only displayed a finely granular matrix. Cytoplasmic droplets have also been observed in the white-backed vulture where they have been described as proximal droplets [28]. Although the location of the avian cytoplasmic droplet is similar to that described for the proximal droplet in mammals [12] and which is viewed as a major defect by Blom [49], there is presently no evidence to suggest that the avian cytoplasmic droplet shifts significantly from its point of origin (proximal droplet) to a more distal location (distal droplet). It remains to be determined whether the detrimental effect of the avian cytoplasmic droplet is purely mechanical in nature, or may reflect a deficiency in sperm maturation. The latter possibility may be suggested by the lack of complete chromatin condensation seen in bent heads associated with cytoplasmic droplets. A detailed study of the process of spermiation will be required to fully appreciate the importance of the avian cytoplasmic droplet.

\subsection{Classification of sperm defects}

Various classification systems have been proposed for the morphological assessment of mammalian sperm $[12,15,49-53]$, and the importance of adopting a uniform (single) system for a specific species has been emphasised [5,54,55]. Earlier classification systems identified primary and secondary sperm abnormalities [56] or divided anomalies into major or minor defects [49]. A more modern concept is that of compensable or uncompensable sperm 
defects [53]. Further examples include the use of 'Strict Atlas Criteria' for assessing normal sperm morphology in humans [15], the identification of genetic sperm defects [50] and the development of a multidimensional system for the recording and interpretation of sperm morphology in bulls [52].

Although criticism of some of the above classification systems has been expressed, each relies on the identification of specific sperm abnormalities and each presents a detailed list of observable anomalies, most of which have been thoroughly described by light microscopy supported by numerous ultrastructural studies. In contrast, the morphological classification of avian sperm abnormalities lacks uniformity and although the presence of abnormal sperm has been reported in a variety of bird species [17,21,26,27,28,35,41,57], the anomalies are described in broad and variable terms which makes it difficult to draw meaningful comparisons. The lack of detailed descriptions of avian sperm abnormalities is compounded, with few exceptions $[19,22,29,30,31]$, by a dearth of supportive ultrastructural data. This situation is clearly illustrated in ratites, where no uniform system for classifying sperm abnormalities has been proposed. Bertschinger et al [6], for example, describe the most common major sperm defects in ostrich sperm as "mid-piece reflexes and coiled tails ('Dag' defects), partial or complete aplasia of the mitochondrial sheath, persistent cytoplasmic droplets and abnormally shaped heads". Other studies on the ostrich simply note the incidence of abnormal sperm without providing descriptions of the defects [24,25]. Malecki et al [23] describe five types of sperm abnormalities in the emu, namely, bent, giant, broken, droplet and distorted, without defining the structural characteristics of the defects.

It should be conceded that the adoption of a single uniform system for the classification of avian sperm defects or even ratite sperm defects is challenging. However, it would appear from the present study and a review of the relevant literature that a system based on the main morphologically identifiable segments of the sperm cell would be the most practical method. Based on observations from the present study, and information in the literature, it is tentatively proposed that the following morphological classification as set out in Table 2 be used to describe and categorise sperm defects in ratites.

As illustrated in Table 2, it is important to agree on a uniform terminology for the description of specific defects. For example, the specific form of head bending referred to as kinking (a single twist of the head) in the present study appears to be described as a 'knotted' head in studies of rooster sperm [35]. More accurate descriptions of avian sperm abnormalities are required to definitively establish, for example, whether knotted heads and kinked heads are indeed synonymous. In the present study no acrosome defects were observed, neither are they mentioned in other studies of ratite sperm defects $[6,23]$. However this should not invalidate the category of acrosome defects when studying ratite sperm as they may very well occur in this group of birds. Likewise, the 'Dag' defect identified by Bertschinger et al [6] in ostrich semen is not specifically identified in other avian studies as a subdivision of either mid-piece or tail abnormalities, although tail coiling has been described [18,35,41]. Again, ultrastructural studies will be required to reveal the specific structural peculiarities that would define a particular form of tail coiling as the 'Dag' defect. There is little doubt that more defects will be added to each of the main categories in this list as further studies are conducted on ratite sperm.

This study attempts to consolidate the disparate descriptions and terminology used to describe abnormal sperm in birds into a uniform system for the recording of ratite sperm defects. The work also underlines the importance of detailed morphological descriptions of abnormal avian sperm as an essential prerequisite for the accurate placement of specific sperm defects in appropriate categories. To our knowledge, this is the first ultrastructural report on emu sperm abnormalities and provides a fresh insight into the structure of certain defects and their possible formation. This information may be of value in the application of reproduction techniques such as AI, both for breeding and raising commercially important birds and the conservation of rare species [2,26,34].

\section{Acknowledgments}

The authors wish to thank Mrs. P. Rough for providing the material, Prof. J. Nöthling for critical appraisal of the manuscript and the University of Pretoria for financial support.

\section{References}

[1] Deeming DC, Ar A. Factors affecting the success of commercial incubation. In: The Ostrich. Biology, Production and Health, Deeming DC (Ed.), CABI Publishing, 1999, pp. 159-90.

[2] Malecki IA, Rybnik PK, Martin GB. Artificial insemination technology for ratites: a review. Aust J Exp Agr 2008;48: 1284-92. 
[3] Soley, JT, Groenewald HB. Reproduction. In: The Ostrich. Biology, Production and Health, Deeming, DC (Ed.), CABI Publishing, 1999, pp. 129-58.

[4] Malecki IA, Beesley J, Martin GB. Changes in the characteristics of emu sperm with season. Brit Poultry Sci Suppl 2000;41: S18.

[5] Malmgren L. Assessing the quality of raw semen: a review. Theriogenology 1997;48:523-30.

[6] Bertschinger HJ, Burger WP, Soley JT, de Lange JH. Semen collection and evaluation of the male ostrich. Proc Biennial Congr S Afr Vet Assoc, Grahamstown, 1992, pp. 154-8.

[7] Asa C, Phillips DM, Stover J. Ultrastructure of spermatozoa of the crested tinamou. J Ultra Mol Struct R 1986;94:170-5.

[8] Baccetti B, Burrini AG, Falchetti E. Spermatozoa and relationships in paleognath birds. Biol Cell 1991;71:209-16.

[9] Phillips DM, Asa CS. Development of spermatozoa in the rhea. Anat Record 1989;223:276-82.

[10] Soley JT. Ultrastructure of ostrich (Struthio camelus) spermatozoa: I. Transmission electron microscopy. Onderstepoort J Vet Res 1993;60:119-30.

[11] Soley JT, Roberts DC. Ultrastructure of ostrich (Struthio camelus) spermatozoa: II. Scanning electron microscopy. Onderstepoort J Vet Res 1994;61:239-46.

[12] Barth AD, Oko RJ. Abnormal Morphology of Bovine Spermatozoa. Iowa State University Press, 1989.

[13] Feito RJ. Ultrastructural study of abnormal spermiogenesis in four hystricognate rodents. J Reprod Fert 1990;88:411-8.

[14] Holstein AF, Roosen-Runge EC, Schirren C. Illustrated Pathology of Human Spermatogenesis. Grosse Verlag, 1988.

[15] Menkveld R, Swanson RJ, Oettlé EE, Acosta AA, Kruger TF, Oehninger S. Atlas of Human Sperm Morphology. Williams \& Wilkins, 1991.

[16] Oettlé EE, Soley JT. Sperm abnormalities in the dog: a light and electron microscopic study. Vet Med Rev 1988;59:28-70.

[17] Łukaszewicz E, Jerysz A, Partyka A, Siudzińska A. Efficacy of evaluation of rooster sperm morphology using different staining methods. Res Vet Sci 2008;85:583-8.

[18] Kamar GAR, Rizik MAA. Semen characteristics of two breeds of turkeys. J Reprod Fert 1972;29:317-25.

[19] Marquez BJ, Ogasawara FX. Scanning electron microscope studies of turkey semen. Poultry Sci 1975;54:1139-43.

[20] Alkan S, Baran A, Özdaş ŐB, Evecen M. Morphological defects in turkey semen. Turk J Vet Anim Sci 2002;26:1087-92.

[21] Penfold LM, Wildt DE, Herzog TL, Lynch W, Ware L, Derrickson SE, Monfort SL. Seasonal patterns of LH, testosterone and semen quality in the Northern pintail duck (Anas acuta). Reprod Fertil Dev 2000;12:229-35.

[22] Ferdinand A. Licht- und elektronenmikroskopische Untersuchungen zur Morphologie von Ganterspermatozoen. Dissertation zur erlangung des grades eines Doctor Medicinae Veterinariae. Hannover, 1992.

[23] Malecki IA, Cummins JM, Martin GB, Lindsay DR. Effect of collection frequency on semen quality and the frequency of abnormal forms of spermatozoa in the emu. P Aus S Anim 1998;22:406.

[24] Hemberger MY, Hospes R, Bostedt H. Semen collection, examination and spermiogram in ostriches. Reprod Domest Anim 2001;36:241-3.

[25] Rozenboim I, Navot A, Snapir N, Rosenstrauch A, El Halawani ME, Gvaryahu G, Degen A. Method for collecting semen from the ostrich (Struthio camelus) and some of its quantitative and qualitative characteristics. Brit Poultry Sci 2003;44:607-11.
[26] Lindsay C, Staines HJ, McCormick P, McCullum C, Choulani F, Wishart GJ. Variability in the size of the nucleus in spermatozoa from the Houbara bustards, Chlamydotis undulate undulate. J Reprod Fert 1999;117:307-13.

[27] Stelzer G, Crosta L, Bürkle M, Krautwald-Junghanns M-E. Attempted semen collection using the massage technique and semen analysis in various psittacine species. J Avian Med Surg 2005;19:7-13.

[28] Umapathy G, Sontakke S, Reddy A, Ahmed S, Shivaji S. Semen characteristics of the captive Indian white-backed vulture. Biol Reprod 2005;73:1039-45.

[29] Soley JT, Els HJ. The ultrastructure of retained cytoplasmic droplets in ostrich spermatozoa. Proc Microsc Soc South Afr 1993;23:58.

[30] Soley JT, Bertschinger HJ, Els HJ, Burger WP. The morphology and incidence of retained cytoplasmic droplets in ostrich spermatozoa. In: Deeming DC (Ed.), Improving our Understanding of Ratites in a Farming Environment. Ratite Conference, Manchester, UK, 1996, pp. 16-8.

[31] Bakst MR, Sexton TJ. Fertilizing capacity and ultrastructure of fowl and turkey spermatozoa before and after freezing. J Reprod Fert 1979;55:1-7.

[32] Jamieson BGM. Avian spermatozoa: Structure and Phylogeny. In: Jamieson BGM (Ed.), Reproductive Biology and Phylogeny of Birds Part A. Science Publishers, 2007, pp. 349-511.

[33] Gee GF, Bertschinger H, Donoghue AM, Blanco J, Soley JT. Reproduction in nondomestic birds: Physiology, semen collection, artificial insemination and cryopreservation. Avian Poult Biol Rev 2004;15:47-101.

[34] Blanco JM, Wildt DE, Höfle U, Voelker W, Donoghue AM. Implementing artificial insemination as an effective tool for $e x$ situ conservation of endangered avian species. Theriogenology 2009;71:200-13.

[35] Tabatabaei S, Batavani RA, Talebi AR. Comparison of semen quality in indigenous and ross broiler breeder roosters. J Anim Vet Adv 2009;8:90-3.

[36] Zini A, Phillips A, Courchesne A, Boman JM, Baazeem A, Bissonnette F, Kadoch IJ, San Gabriel M. Sperm head morphology is related to high deoxyribonucleic acid stainability assessed by sperm chromatin structure assay. Fertil Steril 2009; 91:2495-500.

[37] Bakst MR. Fertilizing capacity and ultrastructure of fowl and turkey spermatozoa in hypotonic extender. J Reprod Fert 1980; 60:121-7.

[38] Soley JT. Nuclear morphogenesis and the role of the manchette during spermiogenesis in the ostrich (Struthio camelus). J Anat 1997; 190:563-76.

[39] Blom E, Birch-Andersen A. Ultrastructure of the decapitated sperm defect in Guernsey bulls. J Reprod Fert 1970;23:67-72.

[40] Chemes HE, Puigdomenech ET, Carizza C, Brugo Olmedo S, Zanchetti F, Hermes R. Acephalic spermatozoa and abnormal development of the head-neck attachment: a human syndrome of genetic origin. Hum Reprod 1999;14:1811-8.

[41] Siudzińska A, Łukaszewicz E. Effect of semen extenders and storage time on sperm morphology of four chicken breeds. J Appl Poultry Res 2008;17:101-8.

[42] Saeki Y. Crooked-ncked spermatozoa in relation to low fertility in the artificial insemination of fowl. Poultry Sci 1960;39: $1354-60$.

[43] Humphreys PN. Brief observations on the semen and spermatozoa of certain passerine and non-passerine birds. J Reprod Fert 1972;29:327-36. 
[44] Blom E. A new sterilizing and hereditary defect (the 'Dag' defect) located in the bull sperm tail. Nature 1966;209:73940.

[45] Zuleta M, Koefoed-Johnsen HH, Bresciani J. The Dag defect of the tail of the bull spermatozoon. Observations with the scanning electron microscope. Copenhagen: Yearbook Royal Vet Agric Univ, 1978, pp. 21-6.

[46] Weissenberg R, Aviram A, Golan R, Lewin LM, Levron J, Madgar I, Dor J, Barkai G, Goldman B. Concurrent use of flow cytometry and fluorescence in-situ hybridization techniques for detecting faulty meiosis in a human sperm sample. Mol Human Reprod 1998;4:61-8.

[47] Kopp C, Sukura A, Tuunainen E, Gustavsson I, Parvinen M, Anderson M. Multinuclear-multiflagellar sperm defect in a bull - a new sterilizing sperm defect. Reprod Dom Anim 2007;42:208-13.

[48] Maretta M. Ultrastructure of double and multiple sperm tails in drakes. Vet Med Czech 1979;24:679-89.

[49] Blom E. The ultrastructure of some characteristic sperm defects and a proposal for a new classification of the bull spermiogram. Atti del VII Simp Int de Zoo., Milano, 1972, pp. 125-39.
[50] Chenoweth PJ. Genetic sperm defects. Theriogenology 2005; 64:457-68.

[51] Mocé E, Graham JK. In vitro evaluation of sperm quality. Anim Reprod Sci 2008;105:104-18.

[52] Nöthling JO, Irons PC. A simple multidimensional system for the recording and interpretation of sperm morphology in bulls. Theriogenology 2008;69:603-11.

[53] Saacke RG, Nadir S, Nebel RL. Relationship of semen quality to sperm transport, fertilization, and embryo quality in ruminants. Theriogenology 1994;41:45-50.

[54] Card C. Cellular associations and the differential spermiogram: Making sense of stallion spermatozoal morphology. Theriogenology 2005;64;558-67.

[55] Graham JK, Mocé E. Fertility evaluation of frozen/thawed semen. Theriogenology 2005;64:492-504.

[56] Blom E. A simple rapid staining method for the differentiation between live and dead sperm cells by means of eosin and nigrosin. Nordisk Veterinaer Medicin 1950;2:58-61.

[57] Klimowicz M, Łukaszewicz E, Dubiel A. Effect of collection frequency on quantitative and qualitative characteristics of pigeon (Columba livia) semen. Brit Poultry Sci 2005;46:361-5. 